\section{Preclinical test for prion diseases}

SIR - The recent recognition that there may be a link between bovine spongiform encephalopathy (BSE) in cattle and Creutzfeldt-Jakob disease (CJD) in humans ${ }^{1}$ highlights the urgent need to find a method for the preclinical diagnosis of transmissible spongiform encephalopathies, or prion diseases. Until then, given the long incubation period of the disease agent, control measures will be hampered. Here we suggest a possible approach to an early preclinical diagnostic method for transmissible spongiform encephalopathies using scrapie in sheep as a model.

Infected animals and humans have neither a disease-specific immune response nor consistent biochemical, haematological and gross pathological abnormalities. The early diagnosis of transmissible spongiform encephalopathies therefore depends on the recognition of clinical signs, electroencephalography or magnetic resonance imaging techniques, or the more invasive method of taking brain biopsies.

Confirmatory diagnosis, however, depends on histological examination of the brain of suspected cases. An altered protein, $\mathrm{PrP}^{\mathrm{Sc}}$, can be detected in the brain of diseased individuals, by immunohistochemistry or other protein-detection

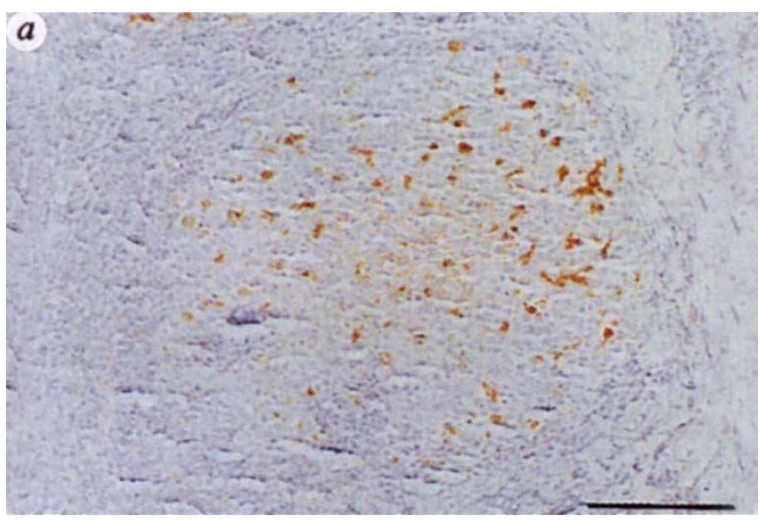

(b)

a, Positive PrP ${ }^{S c}$ immuno-staining in lymphoid follicles in tonsillar biopsy from a scrapie-susceptible sheep ( $\mathrm{PrP}^{\mathrm{VQ} / \mathrm{VQ}}$ ) at age 10 months. Peroxidase-labelled streptavidin-biotin staining. $b$, Negatively staining tonsillar biopsy from a $\operatorname{PrP}^{\mathrm{VQ} / \mathrm{AR}}$ sheep at the same age. Scale bars, $100 \mu \mathrm{m}$. methods. Numerous studies have confirmed $\mathrm{PrP}^{\mathrm{Sc}}$ as a sensitive and specific marker of this group of diseases ${ }^{2}$.

Immunohistochemistry does not demonstrate infectivity, but it does have many advantages over the mouse bioassay, which is considered a more sensitive detection method but far too cumbersome and time-consuming to become a practical diagnostic method. Various tissues blood, urine, tissue fibroblasts and, particularly in animals, lymphoid tissue - have been used in attempts to develop an early-detection technique for these encephalopathies. Most attempts were either negative or inconclusive ${ }^{3}$, but we have built on the classic work of Hadlow, who showed that in sheep naturally infected with scrapie, infectivity was detectable in lymphoid tissue as early as 10-14 months of age, well before it occurred in the central nervous system ${ }^{4,5}$.

Having demonstrated the consistent presence of the scrapie-associated $\mathrm{PrP}^{\mathrm{Sc}}$ in tonsils of a group $(n=55)$ of naturally infected, clinically positive scrapie sheep by immunohistochemistry ${ }^{6}$, using antibodies directed to selected, synthetic PrP-based peptides ${ }^{7}$, we have now detected $\mathrm{PrP}^{\mathrm{Sc}}$ in tonsils of sheep in the preclinical stage of the disease, long before the onset of clinical signs.

We selected a group of ten purposely bred lambs, six of them homozygous for the $\mathrm{PrP}^{\mathrm{VQ}}$ allele (with the residue valine $(\mathrm{V})$ at position 136 of the amino-acid (Q) at position 171). The remaining four lambs were heterozygous, possessing one $\mathrm{PrP}^{\mathrm{VQ}}$ allele and one $\operatorname{PrP}^{\mathrm{AR}}$ allele (with alanine at position 136 and arginine at position 171). In several breeds, this $\operatorname{PrP}^{\mathrm{VQ}}$ allele is significantly associated with an increased susceptibility to scrapie, whereas the $\operatorname{PrP}^{\mathrm{AR}}$ allele is significantly associated with increased resistance of sheep to scrapie ${ }^{8}$.

The ten lambs were born and raised on a farm where scrapie has been occurring for several years. In this flock, we had observed that sheep with the genotype $\mathrm{PrP} \mathrm{P}^{\mathrm{V} / \mathrm{VQ}}$ died from scrapie when about 25 months old, but that most of the sheep with the genotype $\operatorname{Pr} \mathrm{P}^{\mathrm{VQ} / \mathrm{AR}}$ were still healthy at 70 months. Born sequence and glutamine and raised in this environment, the $\mathrm{PrP}^{\mathrm{VQ} / \mathrm{VQ}}$ sheep would thus be expected to develop disease symptoms when about 25 months old, whereas the $\mathrm{PrP}^{\mathrm{VQ} / \mathrm{AR}}$ sheep would stay healthy. We therefore regarded these two groups of sheep as a suitable model for studying changes at known stages of the incubation period.

We took tonsillar biopsies from these animals at approximately 10 (range 9.5-10) months after birth, when none of the sheep showed clinical signs of scrapie. We found clear, extensive $\mathrm{PrP}^{\mathrm{Sc}}$ immunostaining in all six susceptible $\operatorname{PrP}^{\mathrm{VQ} / V Q}$ sheep ( $a$ in the figure) at 10 months of age, but detected no $\mathrm{PrP}^{\mathrm{Sc}}$ immunostaining in any of the resistant $\operatorname{PrP}^{\mathrm{VQ} / \mathrm{AR}}$ sheep ( $b$ in the figure).

We have thus detected scrapie-associated $\mathrm{PrP}^{\mathrm{Sc}}$ in tonsils of sheep at less than half the expected incubation period, approximately one year before the expected onset of clinical disease. We did not detect this $\mathrm{PrP}^{\mathrm{Sc}}$ protein in sheep expected to develop scrapie at a much later stage or to remain healthy throughout their lives. Thus, screening tonsillar tissue for $\operatorname{PrP}^{\mathrm{Sc}}$ by immunohistochemistry offers a possibility of preclinical diagnosis in sheep scrapie.

In view of the negative transmission experiments using different peripheral tissues of cattle affected by $\mathrm{BSE}^{9}$, our technique may not work in cattle. However, because immunohistochemistry has not been tried in depth as a diagnostic test, and because the mouse bioassay may not be sensitive enough in the case of $\mathrm{BSE}$, it is surely worth investigating our suggestion further.

An early diagnosis of BSE could alleviate the draconian measures proposed in the United Kingdom for culling potentially BSE-infected cattle. The technique of taking tonsillar biopsies in live cattle is feasible and would be even easier than in sheep. In human spongiform encephalopathies, this approach has even brighter prospects, as infectivity has been detected in various lymphoid tissues of CJD patients ${ }^{10}$, and tonsil biopsy is a relatively noninvasive, easy technique.

B. E. C. Schreuder, L. J. M. van Keulen

M. E. W. Vromans, J. P. M. Langeveld

M. A. Smits

DLO-Institute for Animal Science and Health (ID-DLO), PO Box 65, 8200 AB Lelystad, The Netherlands

1. Will, R. G. et al. Lancet 374, 921-925 (1996).

2. Prusiner, S., Collinge, J. \& Anderton, B. in Prion Diseases in Humans and Animals (Ellis Horwood, London, 1992)

3. Schreuder, B. E. C. Vet. Q. 16, 174-181 (1994)

4. Hadlow, W. J., Kennedy, R. C. \& Race, R. E. J. infect. Dis. 146, 657-664 (1982).

5. Hadlow, W. J. et al. Vet. Path. 17, 187-199 (1980).

6. van Keulen, L. J. M. et al. J. clin. Microbiol. 34, 1228-1231 (1996)

7. van Keulen, L. J. M. et al. Vet. Path. 32, 299-308 (1995).

8. Belt, P. B. G. M. et al. J. gen. Virol. 76, 509-517 (1995).

9. Fraser, H. \& Foster, J. D. in Proc. Consult. BSE sci. vet. Committee CEC 145-160 (Brussels, September 1993). 10. Brown, P. et al. Ann. Neurol. 35, 513-519 (1994). 